\title{
Elevated Neutrophil-to-Lymphocyte Ratio Predicts Survival in Patients with Advanced Gastric Cancer Treated with Trastuzumab Combination Chemotherapy
}

\author{
GI YONG HWANG ${ }^{1,2^{*}}$, DONG WON BAEK ${ }^{1,2^{*}}$, HEE JEONG CHO ${ }^{1,2}$, SOO JUNG LEE ${ }^{1,2}$, YEE SOO CHAE ${ }^{1,2}$, \\ BYUNG WOOG KANG ${ }^{1,2}$, IN HEE LEE ${ }^{1,2}$, JONG GWANG KIM ${ }^{1,2}$, AN NA SEO ${ }^{2,3}$, HAN IK BAE HA $^{2,3}$ \\ KI BUM PARK ${ }^{2,4}$, JI YEON PARK ${ }^{2,4}$, OH KYOUNG KWON ${ }^{2,4}$, SEUNG SOO LEE ${ }^{2,4}$ and HO YOUNG CHUNG ${ }^{2,4}$ \\ ${ }^{1}$ Department of Oncology/Hematology, School of Medicine, Kyungpook National University, \\ Kyungpook National University Cancer Research Institute, Daegu, Republic of Korea; \\ ${ }^{2}$ Kyungpook National University Hospital, Daegu, Republic of Korea; \\ ${ }^{3}$ Department of Pathology, School of Medicine, Kyungpook National University, Daegu, Republic of Korea; \\ ${ }^{4}$ Department of Surgery, School of Medicine, Kyungpook National University, Daegu, Republic of Korea
}

\begin{abstract}
Background/Aim: This study investigated the clinical prognostic relevance of the neutrophil-to-lymphocyte ratio $(N L R)$ in patients with human epidermal receptor 2 (HER2)-positive metastatic advanced gastric cancer (AGC) treated with combination chemotherapy including trastuzumab. Patients and Methods: This is a retrospective analysis of 73 patients diagnosed with metastatic AGC who were treated with trastuzumab combination chemotherapy. NLR was calculated as the neutrophil count divided by the lymphocyte count. A cutoff value of 3 was selected, which classified patients into two categories, low ( $\leq 3.0)$ or high (>3.0). Results: In the univariate analysis, the high-NLR patients showed a significantly shorter progression-free survival (PFS) and overall survival (OS) than the low-NLR patients (PFS, $p=0.012$, OS, $p=0.047$ ). In the multivariate analysis, the high NLR was independently associated with a shorter PFS $(p=0.015)$ and OS $(p=0.040)$. Conclusion: This study found that a high NLR was associated with a shorter PFS and OS in patients with HER2-positive gastric cancer treated with trastuzumab.
\end{abstract}

\footnotetext{
*These Authors contributed equally to this work.

Correspondence to: Byung Woog Kang, Department of Oncology/Hematology, School of Medicine, Kyungpook National University, 807 Hogukno, Buk-gu, Daegu 41404, Republic of Korea. Tel: +82 532002622, Fax: +82 532002029, e-mail: bwkang@knu.ac.kr; Oh Kyoung Kwon, Department of Surgery, School of Medicine, Kyungpook National University, 807 Hogukno, Buk-gu, Daegu 41404, Republic of Korea. Tel: +82 532002622, Fax: +82 532002027, e-mail: kok007@hanmail.net
}

Key Words: Gastric cancer, trastuzumab, neutrophil-to-lymphocyte ratio, prognostic factor.
At the time of diagnosis, the majority of patients with gastric cancer are in an advanced or inoperable stage, meaning that one of the most important treatment modalities is systemic chemotherapy. The current standard practice for treating advanced gastric cancer (AGC) is a doublet combination regimen of either platinum-based or 5-fluorouracil (5FU)based chemotherapy (1). Also, various new chemotherapeutic agents, including oral $5 \mathrm{FU}$, irinotecan, and taxanes, have been identified to improve the outcomes for AGC (2). A recent targeted-agent trial showed that the addition of trastuzumab to conventional chemotherapy significantly improved the survival of patients with human epidermal receptor 2 (HER2)-positive AGC (3). However, most patients only responded temporarily to the initial trastuzumab chemotherapy and subsequently experienced disease progression. Moreover, the effect of trastuzumab combined chemotherapy would seem to vary depending on systemic and tumor-related factors. Thus, a clinical predictor is needed for HER2-positive metastatic AGC patients treated with trastuzumab.

The prognostic factors for cancer patients can generally be classified into four categories, including patient-related factors, tumor-related factors, systemic inflammatory factors, and treatment-related factors (4). Among these, the systemic inflammatory response and tumor microenvironment are known to play a crucial role in tumor growth, invasion, and metastases $(5,6)$. Also, there is increasing evidence that the neutrophil-to-lymphocyte ratio (NLR) can be an effective prognostic indicator for various malignant tumors (7-15). Several recent studies identified an elevated NLR as predicative of a worse prognosis for patients with gastric cancer (16-21), which was also confirmed by the results of a meta-analysis, where the outcomes for 5,431 patients in 19 
articles revealed the prognostic significance of the pretreatment NLR on overall survival (OS) and progressionfree survival (PFS) in patients with gastric cancer (22). Interestingly, the NLR can affect the treatment outcome by regulating the immune reaction in patients who receive trastuzumab-combined chemotherapy. Notwithstanding, the prognostic role of the NLR in gastric cancer remains controversial, including in HER2-positive metastatic AGC.

Accordingly, this study investigated the prognostic significance of the NLR in patients with HER2-positive metastatic AGC treated with trastuzumab.

\section{Patients and Methods}

Patients and treatment. This study retrospectively identified 73 patients who were diagnosed with metastatic AGC and received trastuzumab combination chemotherapy at Kyungpook National University Chilgok Hospital between May 2011 and August 2017. The inclusion criteria were as follows: stage IV gastric cancer (7th of AJCC) (23); diagnosed with gastric adenocarcinoma based on biopsy; HER2 status of $3+$ for immunohistochemistry or $2+$ for immunohistochemistry and FISH/SISH+. The patient records were also reviewed for data on their medical history, age, sex, backbone chemotherapy regimen, surgical methods, and pathologic results. Patients were excluded if their pathologic or laboratory results were missing or they were lost to follow-up. The study was approved by the Institutional Review Board at Kyungpook National University Hospital. Trastuzumab was administered by intravenous infusion at a dose of $8 \mathrm{mg} / \mathrm{kg}$ on day 1 of the first cycle, followed by $6 \mathrm{mg} / \mathrm{kg}$ every 3 weeks, while capecitabine $1000 \mathrm{mg} / \mathrm{m}^{2}$ was given orally twice a day for 14 days, followed by a 1 -week rest until disease progression or unacceptable toxicity. Sixty to $80 \mathrm{mg} / \mathrm{m}^{2}$ of cisplatin was given on day 1 every 3 weeks for six cycles.

Ethical approval. All procedures performed in studies involving human participants were in accordance with the ethical standards of the institutional and/or national research committee and with the 1964 Declaration of Helsinki and its later amendments or comparable ethical standards.

Informed consent. Informed consent was obtained from all individual participants included in the study.

Definition of laboratory parameters. Blood samples were obtained from the patients before chemotherapy. The NLR was calculated as the neutrophil count divided by the lymphocyte count. A cut-off value of 3 was selected based on a receiver-operating curve (ROC) analysis, and the patients were classified as either low $(\leq 3.0)$ or high $(>3.0)$.

Statistical analysis. PFS was estimated from the time of the commencing treatment to disease progression or death. OS was calculated from the date of diagnosis to death from any cause. The response was evaluated according to the response evaluation criteria in solid tumors (RECIST) version 1.1. Chi-square test and Fisher's exact test were both applied to the qualitative variables. Using ROC curve analysis for predicting events, several candidate cut-off values were tested and the cut-off value representing the maximum $\chi^{2}$ was selected (24). The survival analysis used the Kaplan-Meier method with a log-rank test. A multivariate analysis
Table I. Baseline of patient characteristics.

\begin{tabular}{lc}
\hline Characteristic & Total $(\mathrm{n}=73)$ \\
& $\mathrm{n}(\%)$ \\
\hline Age, years & \\
$\quad$ Median (Range) & $63.0(20.0-87.0)$ \\
Gender & \\
Male & $61(83.6)$ \\
Female & $12(16.4)$ \\
ECOG performance status & \\
0 or 1 & $69(94.5)$ \\
2 & $4(5.5)$ \\
Histologic differentiation & \\
Well differentiated & $2(2.7)$ \\
Moderate differentiated & $39(53.4)$ \\
Poorly differentiated & $25(34.2)$ \\
thers & $6(9.7)$ \\
Number of metastases & \\
1 & $42(57.5)$ \\
2 & $17(23.3)$ \\
$\geq 3$ & $14(19.2)$ \\
Sites of metastases & \\
Liver & $45(61.6)$ \\
Lung & $4(5.5)$ \\
Distant lymph node & $33(45.2)$ \\
Peritoneal seedings & $18(24.7)$ \\
Previous history of surgical resection & \\
Curative & $12(16.4)$ \\
Palliative & $5(6.8)$ \\
Not done & $56(76.8)$ \\
CEA & \\
$<7.0$ & $39(53.4)$ \\
$\geq 7.0$ & $34(46.6)$ \\
Neutrophil-to-lymphocyte ratio & \\
$\leq 3.0$ & $33(45.2)$ \\
$>3.0$ & $40(54.8)$ \\
\hline & \\
\hline & \\
& \\
&
\end{tabular}

was performed using variables with a value of $p<0.1$ in a univariate analysis using Cox's proportional hazards model to derive a potentially suitable set of predictors. Two-sided $p$-values of $<0.05$ were considered to be significant. The statistical analyses were performed using SPSS software version18.0 (SPSS, Inc., Chicago, IL, USA).

\section{Results}

Patient characteristics. The patient characteristics are summarized in Table I. The median age was 63 years (range $=20-87$ years) and the ratio of males to females was approximately $5: 1$. Most of the patients had an ECOG performance status of 0 or $1(94.5 \%)$. The histologic differentiations were as follows: well differentiated $(n=2$, $2.7 \%)$, moderately differentiated $(\mathrm{n}=39,53.4 \%)$ and poorly differentiated $(n=25,34.2 \%)$. The liver $(n=45,61.6 \%)$ and distant lymph nodes $(n=33,45.2 \%)$ were the most common 

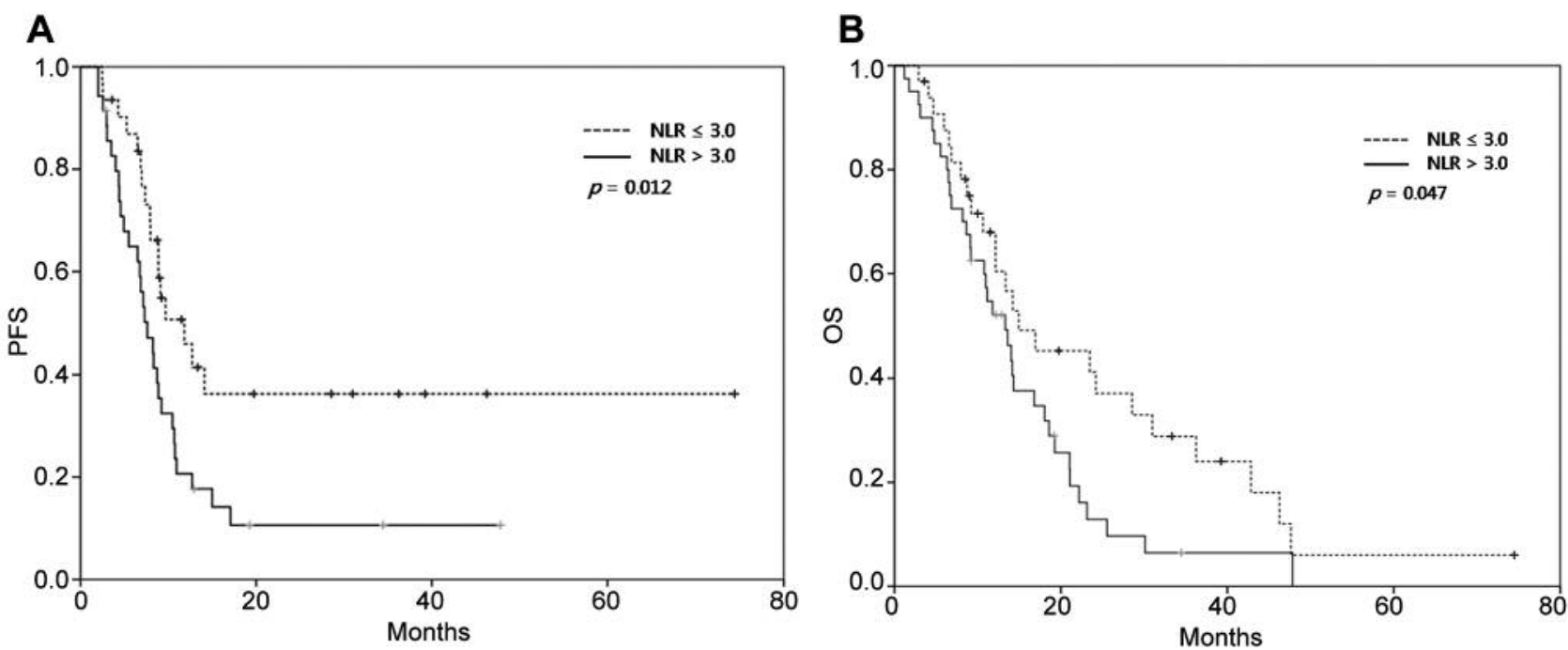

Figure 1. Kaplan-Meier survival curves for (A) progression-free survival (PFS) and (B) overall survival (OS) of 73 patients according to neutrophilto-lymphocyte ratio.

sites of metastases. Before chemotherapy, $12(16.4 \%)$ patients underwent curative surgical resection, and $5(6.8 \%)$ underwent palliative surgical resection. Among the 73 patients, $40(54.8 \%)$ exhibited a high pretreatment NLR $(>3.0)$ and 34 showed a high pretreatment CEA $(\geq 7.0)$.

NLR and response to chemotherapy. The association between NLR and the response rate to chemotherapy is shown in Table II. The clinical outcomes for the high NLR (>3.0) group included: complete response $(n=3,7.5 \%)$, partial response $(n=19,47.5 \%)$, stable disease $(n=7,17.5 \%)$, and progressive disease $(n=9,22.5 \%)$, while the outcomes for the low NLR $(\leq 3.0)$ group included: complete response $(n=4,12 \%)$, partial response $(n=20,61 \%)$, stable disease $(n=7,21 \%)$, and progressive disease $(n=0,0 \%)$. Thus, a high pretreatment NLR was significantly associated with poor clinical outcomes when compared with the low NLR group $(p=0.038)$.

NLR and survival outcome. At the time of the last follow-up, the median follow-up duration was 12.1 months (range=1.274.5 months). During the analyses, 47 (64\%) patients experienced recurrence and $59(81 \%)$ patients died. In the univariate analysis, the high NLR patients showed a significantly shorter PFS and OS than the low NLR patients (PFS, $p=0.012$, OS, $p=0.047$, Figure 1). In the multivariate analysis using a Cox proportional hazard model adjusted for age, histologic differentiation, and CEA, a high NLR was independently associated with a shorter PFS $(\mathrm{HR}=2.113$, $95 \% \mathrm{CI}=1.155-3.866, p=0.015)$ and $\mathrm{OS} \quad(\mathrm{HR}=1.795$, 95\% CI $=1.026-3.140, p=0.040$ ) (Table III).
Table II. The association between neutrophil-to-lymphocyte ratio and response rate.

\begin{tabular}{lcc}
\hline & $\begin{array}{c}\text { NLR } \leq 3.0(\mathrm{n}=33) \\
\mathrm{n}(\%)\end{array}$ & $\begin{array}{c}\text { NLR }>3.0(\mathrm{n}=40) \\
\mathrm{n}(\%)\end{array}$ \\
\hline Complete response & $4(12 \%)$ & $3(7.5 \%)$ \\
Partial response & $20(61 \%)$ & $19(47.5 \%)$ \\
Stable disease & $7(21 \%)$ & $7(17.5 \%)$ \\
Progressive disease & $0(0 \%)$ & $9(22.5 \%)$ \\
Not evaluable & $2(6 \%)$ & $2(5 \%)$ \\
\hline
\end{tabular}

NLR: Neutrophil-to-lymphocyte ratio.

\section{Discussion}

When investigating the clinical significance of the NLR in 73 patients with HER2-positive AGC treated with trastuzumab, the NLR was identified as an independent prognostic factor of a worse PFS and OS. Consequently, a high NLR would seem to play an important role in aggravating tumor progression in patients with HER2-postivie AGC possibly by altering the inflammatory response to cancer.

Neutrophils are produced in the bone marrow through a series of progenitors, from immature stem cells and granulocyte-macrophage progenitors to committed progenitors that finally generate mature neutrophils (25). Several transcription factors and cytokines are related with this process of proliferation and differentiation at different stages. Neutrophils are a type of phagocytes normally found in the 
Table III. Multivariate analyses for progression-free survival and overall survival.

\begin{tabular}{|c|c|c|c|c|c|c|c|}
\hline \multirow[t]{2}{*}{ Variables } & \multirow[t]{2}{*}{ Category } & \multicolumn{3}{|c|}{ Progression-free survival } & \multicolumn{3}{|c|}{ Overall survival } \\
\hline & & $p$-Value & HR & $95 \% \mathrm{CI}$ & $p$-Value & HR & $95 \% \mathrm{CI}$ \\
\hline Age, years & $<63$ vs. $\geq 63$ & 0.287 & 0.728 & $0.406-1.305$ & 0.786 & 0.929 & $0.545-1.583$ \\
\hline Histologic differentiation & WD vs. MD vs. PD \& others & 0.648 & 0.872 & $0.484-1.571$ & 0.516 & 0.834 & $0.482-1.442$ \\
\hline CEA & $<7.0 v s . \geq 7.0$ & 0.101 & 1.642 & $0.908-2.969$ & 0.324 & 1.312 & $0.765-2.248$ \\
\hline NLR & $\leq 3.0 \mathrm{vs} .>3.0$ & 0.015 & 2.113 & $1.155-3.866$ & 0.040 & 1.795 & $1.026-3.140$ \\
\hline
\end{tabular}

WD: Well differentiated; MD: moderate differentiated; PD: poorly differentiated; NLR: neutrophil-to-lymphocyte ratio.

bloodstream during the beginning acute phase of inflammation, particularly as a result of bacterial infection, environmental exposure, and some cancers (26). The role of neutrophils in cancer is complex and multifactorial (27). Neutrophils can act as tumor-promoting leukocytes by altering adhesion molecules, releasing reactive oxygen species, and inducing angiogenesis. In addition, neutrophils can motivate metastatic spreading by inhibiting the natural killer function and facilitating the extravasation of tumor cells (28). In contrast, lymphocytes are generally associated with a good prognosis for gastric cancer (29). In a previous study, the current authors showed an independent association between tumor-infiltrating lymphocytes and favorable outcomes in the case of Epstein-Barr virus-associated gastric cancer (30). Given this context, elevated numbers of neutrophils and decreased numbers of lymphocytes in peripheral blood, as reflected by the NLR, can be reasonably associated with the clinical outcomes of gastric cancer. An elevated NLR has already been correlated with a poor prognosis in patients with various malignant tumors (31). Similarly, the current study showed an association between an elevated NLR and poorer survival outcomes in the case of AGC.

The Cancer Genome Atlas Research Network recently produced a comprehensive molecular characterization of 295 gastric cancers using various platforms and indicated that EBV-positive tumors and microsatellite unstable tumors often show immune cell signaling activation (32). Furthermore, the recent ONO-4538-12 trial, which compared nivolumab, a human IgG4 anti-PD-1 monoclonal antibody, to a placebo in patients with unresectable advanced or recurrent gastric cancer, also showed a significantly prolonged OS for the nivolumab arm (33). Thus, for gastric cancers, these findings provide strong evidence that immune cells contribute to determining the prognosis (29). Several clinical studies have already reported unfavorable outcomes for AGC patients with the high NLR. Gonda et al. evaluated the correlation between the NLR and survival in 110 patients with AGC receiving S1 plus cisplatin (24). They found that the patients with a NLR $>3.0$ had a shorter OS than those with a NLR $<3.0$, which is consistent with the current results in terms of the survival outcomes and cutoff. Another study also reported that a high NLR was significantly correlated with age, tumor size, depth of invasion, lymphatic involvement, venous involvement, and disease stage (20). This multivariate analysis also identified the c-reactive protein/albumin ratio and NLR as a combined independent prognostic indicator. Moreover, a recent metaanalysis showed an association between a high NLR and worse long-term outcomes in gastric cancer (22). An elevated NLR was associated with a significantly poorer OS and PFS when compared with patients with normal NLR. Notwithstanding, these similar prognostic results may have been influenced by using the same NLR cutoff value of 3.00. Plus, several additional factors should also be taken into account, including ethnicity, cultural challenges, different genetic structures, several inflammatory statuses, and other pathological factors (34).

Trastuzumab binds to the juxtamembrane portion of the extracellular domain of the HER2 receptor, thereby preventing the activation of its intracellular tyrosine kinase (35). Preclinical models have suggested that trastuzumab recruits immune effector cells that are responsible for antibody-dependent cytotoxicity and inhibits angiogenesis by modulating pro-angiogenic and anti-angiogenic factors (36, 37). These findings also support the idea that the NLR can affect the treatment outcome by regulating the immune reaction in patients who receive trastuzumab combined chemotherapy. Some target agents can boost the host immune response and change the activity of the immune system (27). For instance, an elevated NLR is an adverse prognostic factor for metastatic renal cell carcinoma treated with vascular endothelial growth factor-targeted agents (38). Furthermore, several studies have already associated the NLR with a poor OS in patients with HER2-positive breast cancer. Therefore, the current results can help in understanding the role of the NLR in the outcome of HER2-positive gastric cancer when treated with trastuzumab chemotherapy.

Although the present data identified a significant prognostic role for the NLR in HER2-positive gastric cancer treated with trastuzumab, these results should be interpreted carefully. First, a standard cut-off value for the NLR has not yet been 
clearly established. Second, since the level of neutrophils and lymphocytes is measured in the blood at a single point in time, there is no reflection of any dynamic changes of neutrophils and lymphocytes. Finally, although the current study had a homogeneous ethnic identity and equivalent treatment application, there was a potential limitation as it was a retrospective evaluation. Consequently, further large-scale studies are required to validate the current results.

In conclusion, the NLR was shown to be associated with the prognosis of patients with HER2-positive gastric cancer treated with trastuzumab. For the current patient cohort, the results also revealed that a high NLR was independently associated with a shorter PFS and OS. Thus, the NLR could be a prognostic factor for predicting the oncological outcomes of patients with HER2-positive gastric cancer.

\section{Conflicts of Interest}

The Authors declare that they have no conflicts of interest.

\section{Acknowledgements}

This work was supported by the National Research Foundation of Korea (NRF) Grant funded by the Korea government (2014R1A5A2009242) and Basic Science Research Program through the National Research Foundation of Korea (NRF) funded by the Ministry of Education (2017R1D1A3B03032320).

\section{References}

1 Price TJ, Shapiro JD, Segelov E, Karapetis CS, Pavlakis N, Van Cutsem E, Shah MA, Kang YK and Tebbutt NC: Management of advanced gastric cancer. Expert Rev Gastroenterol Hepatol 6: 199-208, 2012.

2 Kang BW, Kim JG, Kwon OK, Chung HY and Yu W: Nonplatinum-based chemotherapy for treatment of advanced gastric cancer: 5-fluorouracil, taxanes, and irinotecan. World J Gastroenterol 20: 5396-5402, 2014.

3 Bang YJ, Van Cutsem E, Feyereislova A, Chung HC, Shen L, Sawaki A, Lordick F, Ohtsu A, Omuro Y, Satoh T, Aprile G, Kulikov E, Hill J, Lehle M, Ruschoff J, Kang YK and To GATI: Trastuzumab in combination with chemotherapy versus chemotherapy alone for treatment of HER2-positive advanced gastric or gastro-oesophageal junction cancer (ToGA): a phase 3 , open-label, randomised controlled trial. Lancet 376: 687-697, 2010.

4 Verdaguer H, Sauri T and Macarulla T: Predictive and prognostic biomarkers in personalized gastrointestinal cancer treatment. J Gastrointest Oncol 8: 405-417, 2017.

5 Aggarwal BB, Vijayalekshmi RV and Sung B: Targeting inflammatory pathways for prevention and therapy of cancer: shortterm friend, long-term foe. Clin Cancer Res 15: 425-430, 2009.

6 Grivennikov SI, Greten FR, and Karin M: Immunity, inflammation, and cancer. Cell 140: 883-899, 2010.

7 Chen S, Zhang L, Yan G, Cheng S, Fathy AH, Yan N and Zhao Y: Neutrophil-to-Lymphocyte Ratio Is a Potential Prognostic Biomarker in Patients with Ovarian Cancer: A Meta-Analysis. Biomed Res Int 2017: 7943467, 2017.
8 Chen Y, Chen K, Xiao X, Nie Y, Qu S, Gong C, Su F and Song E: Pretreatment neutrophil-to-lymphocyte ratio is correlated with response to neoadjuvant chemotherapy as an independent prognostic indicator in breast cancer patients: a retrospective study. BMC Cancer 16: 320, 2016.

9 Haram A, Boland MR, Kelly ME, Bolger JC, Waldron RM and Kerin MJ: The prognostic value of neutrophil-to-lymphocyte ratio in colorectal cancer: A systematic review. J Surg Oncol 115: 470-479, 2017.

10 Lee IH, Hwang S, Lee SJ, Kang BW, Baek D, Kim HJ, Park SY, Park JS, Choi GS, Kim JC, Cho SH and Kim JG: Systemic inflammatory response after preoperative chemoradiotherapy can affect oncologic outcomes in locally advanced rectal cancer. Anticancer Res 37: 1459-1465, 2017.

11 Peng B, Wang YH, Liu YM and Ma LX: Prognostic significance of the neutrophil to lymphocyte ratio in patients with non-small cell lung cancer: a systemic review and meta-analysis. Int J Clin Exp Med 8: 3098-3106, 2015.

12 Saqib R, Pathak S, Smart N, Nunes Q, Rees J, Finch Jones M and Poston G: Prognostic significance of pre-operative inflammatory markers in resected gallbladder cancer: a systematic review. ANZ J Surg, 2017. doi: 10.1111/ans.14300. [Epub ahead of print]

13 Tang X, Du P and Yang Y: The clinical use of neutrophil-tolymphocyte ratio in bladder cancer patients: a systematic review and meta-analysis. Int J Clin Oncol 22: 817-825, 2017.

14 Haruma T, Nakamura K, Nishida T, Ogawa C, Kusumoto T, Seki $\mathrm{N}$ and Hiramatsu Y: Pre-treatment neutrophil to lymphocyte ratio is a predictor of prognosis in endometrial cancer. Anticancer Res 35: 337-343, 2015.

15 Tang X, Wang S, An C, Du P and Yang Y: Preoperative high neutrophil-to-lymphocyte ratio is associated with high-grade bladder cancer. Anticancer Res 37: 4659-4663, 2017.

16 Mimatsu K, Fukino N, Ogasawara Y, Saino Y and Oida T: Utility of inflammatory marker- and nutritional status-based prognostic factors for predicting the prognosis of stage IV gastric cancer patients undergoing non-curative surgery. Anticancer Res 37: 4215-4222, 2017.

17 Gong W, Zhao L, Dong Z, Dou Y, Liu Y, Ma C and Qu X: After neoadjuvant chemotherapy platelet/lymphocyte ratios negatively correlate with prognosis in gastric cancer patients. J Clin Lab Anal, 2017. doi: 10.1002/jcla.22364. [Epub ahead of print]

18 Li S, Lan X, Gao H, Li Z, Chen L, Wang W, Song S, Wang Y, Li C, Zhang $\mathrm{H}$ and Xue Y: Systemic Inflammation Response Index (SIRI), cancer stem cells and survival of localised gastric adenocarcinoma after curative resection. J Cancer Res Clin Oncol 143: 2455-2468, 2017.

19 Miyatani K, Saito H, Kono Y, Murakami Y, Kuroda H, Matsunaga T, Fukumoto Y, Osaki T, Ashida K and Fujiwara Y: Combined analysis of the pre- and postoperative neutrophillymphocyte ratio predicts the outcomes of patients with gastric cancer. Surg Today 48: 300-307, 2018.

20 Saito H, Kono Y, Murakami Y, Shishido Y, Kuroda H, Matsunaga T, Fukumoto Y, Osaki T, Ashida K and Fujiwara Y: Prognostic significance of the preoperative ratio of c-reactive protein to albumin and neutrophil-lymphocyte ratio in gastric cancer patients. World J Surg, 2017. doi: 10.1007/s00268-0174400-1. [Epub ahead of print]

21 Song S, Li C, Li S, Gao H, Lan X and Xue Y: Derived neutrophil to lymphocyte ratio and monocyte to lymphocyte 
ratio may be better biomarkers for predicting overall survival of patients with advanced gastric cancer. Onco Targets Ther 10: 3145-3154, 2017.

22 Sun J, Chen X, Gao P, Song Y, Huang X, Yang Y, Zhao J, Ma $\mathrm{B}$, Gao $\mathrm{X}$ and Wang $\mathrm{Z}$ : can the neutrophil to lymphocyte ratio be used to determine gastric cancer treatment outcomes? A systematic review and meta-analysis. Dis Markers 2016: 7862469, 2016.

23 Edge SB and Compton CC: The American Joint Committee on Cancer: the 7th edition of the AJCC cancer staging manual and the future of TNM. Ann Surg Oncol 17: 1471-1474, 2010

24 Gonda K, Shibata M, Sato Y, Washio M, Takeshita H, Shigeta H, Ogura M, Oka S and Sakuramoto S: Elevated neutrophil-tolymphocyte ratio is associated with nutritional impairment, immune suppression, resistance to S-1 plus cisplatin, and poor prognosis in patients with stage IV gastric cancer. Mol Clin Oncol 7: 1073-1078, 2017.

25 Nicolas-Avila JA, Adrover JM and Hidalgo A: Neutrophils in homeostasis, immunity, and cancer. Immunity 46: 15-28, 2017.

26 Jackaman C, Tomay F, Duong L, Abdol Razak NB, Pixley FJ, Metharom P and Nelson DJ: Aging and cancer: The role of macrophages and neutrophils. Ageing Res Rev 36: 105-116, 2017.

27 Ocana A, Nieto-Jimenez C, Pandiella A and Templeton AJ: Neutrophils in cancer: prognostic role and therapeutic strategies. Mol Cancer 16: 137, 2017.

28 Spiegel A, Brooks MW, Houshyar S, Reinhardt F, Ardolino M, Fessler E, Chen MB, Krall JA, DeCock J, Zervantonakis IK, Iannello A, Iwamoto Y, Cortez-Retamozo V, Kamm RD, Pittet MJ, Raulet DH and Weinberg RA: Neutrophils suppress intraluminal NK cell-mediated tumor cell clearance and enhance extravasation of disseminated carcinoma cells. Cancer Discov 6: 630-649, 2016.

29 Chang WJ, Du Y, Zhao X, Ma LY and Cao GW: Inflammationrelated factors predicting prognosis of gastric cancer. World $\mathrm{J}$ Gastroenterol 20: 4586-4596, 2014.

30 Kang BW, Seo AN, Yoon S, Bae HI, Jeon SW, Kwon OK, Chung HY, Yu W, Kang H and Kim JG: Prognostic value of tumor-infiltrating lymphocytes in Epstein-Barr virus-associated gastric cancer. Ann Oncol 27: 494-501, 2016.

31 Templeton AJ, Ace O, McNamara MG, Al-Mubarak M, VeraBadillo FE, Hermanns T, Seruga B, Ocana A, Tannock IF and Amir E: Prognostic role of platelet to lymphocyte ratio in solid tumors: a systematic review and meta-analysis. Cancer Epidemiol Biomarkers Prev 23: 1204-1212, 2014.
32 Cancer Genome Atlas Research N: Comprehensive molecular characterization of gastric adenocarcinoma. Nature 513: 202209, 2014.

33 Kang YK, Boku N, Satoh T, Ryu MH, Chao Y, Kato K, Chung HC, Chen JS, Muro K, Kang WK, Yeh KH, Yoshikawa T, Oh SC, Bai LY, Tamura T, Lee KW, Hamamoto Y, Kim JG, Chin K, Oh DY, Minashi K, Cho JY, Tsuda M and Chen LT: Nivolumab in patients with advanced gastric or gastro-oesophageal junction cancer refractory to, or intolerant of, at least two previous chemotherapy regimens (ONO-4538-12, ATTRACTION-2): a randomised, double-blind, placebo-controlled, phase 3 trial. Lancet 390: 2461-2471, 2017.

34 Faria SS, Fernandes PC, Jr., Silva MJ, Lima VC, Fontes W, Freitas-Junior R, Eterovic AK and Forget P: The neutrophil-tolymphocyte ratio: a narrative review. Ecancermedicalscience 10: 702, 2016.

35 Hudis CA: Trastuzumab--mechanism of action and use in clinical practice. N Engl J Med 357: 39-51, 2007.

36 Petit AM, Rak J, Hung MC, Rockwell P, Goldstein N, Fendly B and Kerbel RS: Neutralizing antibodies against epidermal growth factor and ErbB-2/neu receptor tyrosine kinases downregulate vascular endothelial growth factor production by tumor cells in vitro and in vivo: angiogenic implications for signal transduction therapy of solid tumors. Am J Pathol 151: 15231530, 1997.

37 Weiner LM and Adams GP: New approaches to antibody therapy. Oncogene 19: 6144-6151, 2000.

38 Heng DY, Xie W, Regan MM, Warren MA, Golshayan AR, Sahi C, Eigl BJ, Ruether JD, Cheng T, North S, Venner P, Knox JJ, Chi KN, Kollmannsberger C, McDermott DF, Oh WK, Atkins MB, Bukowski RM, Rini BI and Choueiri TK: Prognostic factors for overall survival in patients with metastatic renal cell carcinoma treated with vascular endothelial growth factortargeted agents: results from a large, multicenter study. J Clin Oncol 27: 5794-5799, 2009.
Received February 20, 2018

Revised March 6, 2018 Accepted March 7, 2018 